\title{
Automatização do processo de questionários na plataforma LimeSurvey utilizando aprendizagem de máquina
}

\author{
Igor A. Campos*. Leonardo D. Tavares* \\ *Faculdade Cotemig, Rua Santa Cruz, 546. Bairro Barroca, \\ Belo Horizonte/MG. (Tel: (31)3371.3051; e-mail: campos.ia@outlook.com, tavares@cotemig.com.br).
}

\begin{abstract}
Market research dates to the early 20s and currently the online market research market has been increasingly targeted by companies in view of low cost and speed of production and delivery of content. This study aims to present a tool that integrates machine learning in online forms using building automation based on the LimeSurvey platform.
\end{abstract}

Resumo: A pesquisa de mercado data do início da década de 1920 e, atualmente, o mercado de pesquisa de mercado online tem sido cada vez mais visado pelas empresas em vista do baixo custo e agilidade da produção e entrega do conteúdo. $\mathrm{O}$ presente trabalho tem por objetivo apresentar uma ferramenta que integra aprendizagem de máquina na automatização de construção de formulários online utilizando como base a plataforma LimeSurvey.

Keywords: Online Survey; Machine Learning; Market research; Automation; Software

Palavras-chaves: Questionários online, Aprendizagem de máquina; Pesquisa de mercado; Automação; Software

\section{INTRODUÇÃO}

A pesquisa de mercado data do início da década de 1920 , sendo fortemente ampliadas e pesquisada (Jansson-Boyd (2010)). Com o advento da internet o mercado de pesquisa se transformou tirando as limitações de tempo, custo e distância, possuindo um acesso mundial praticamente instantâneo (Freitas, Janissek-Muniz, Moscarola (2004)). O mercado de pesquisa de mercado online tem sido cada vez mais visado pelas empresas em vista do baixo custo e agilidade da produção e entrega do conteúdo acordado.

O software desenvolvido para o presente trabalho tem por objetivo a utilização de técnicas de aprendizagem de máquina para a automatização do processo de criação de enquetes e formulários online, visando a diminuição de erros humanos durante esta etapa e, ainda, o aumento da produtividade e assertividade das questões e campos dos formulários. Com isso espera-se que os questionários sejam respondidos de maneira mais acurada.

Para que este objetivo possa ser atingido foi utilizada a plataforma LimeSurvey ${ }^{l}$ que se trata de um software livre para aplicação de questionários online.

O presente trabalho está organizado como se segue: na Seção 2 é apresentada a etapa de contextualização do problema e a solução proposta com a integração do uso das ferramentas de aprendizagem de máquina. Na Seção 3 são apresentados os resultados encontrados e os modelos de aprendizagem de máquina utilizados. Por fim, na Seção 4 são apresentadas as conclusões e considerações finais.

\section{CONTEXTUALIZAÇÃO}

\subsection{Processo de criação de enquetes utilizando o LimeSurvey}

O solução proposta no presente trabalho utiliza como base a plataforma LimeSurvey para criação de enquetes online, o qual possui uma API (Application Programming Interface) disponível para realização das (criação, disponibilização, encerramento, resultado, entre outras funcionalidades). $\mathrm{O}$ primeiro passo para o desenvolvimento do software foi especificar qual era o procedimento atualmente usado por uma empresa para criar as enquetes e realizar uma análise sobre como aprimorá-lo.

Foi diagnosticado que a criação da enquete tradicionalmente segue os seguintes passos:

1. Venda do serviço de pesquisa online: onde é acordado o preço e como será executado o projeto;

2. Elaboração do documento questionário: que pode ser realizada pelo cliente ou pela empresa contratada. Esta etapa gera um documento que contém todas as perguntas e métodos de entrada para as respostas;

3. Validação do documento: as partes interessadas do projeto validam a viabilidade da criação e finalizam o escopo do projeto;

\footnotetext{
${ }^{1}$ Disponível em: www.limesurvey.org
} 
4. Programação do questionário: um programador é designado para criar a enquete, recebendo o documento e tendo que cadastrar as perguntas e respostas assim como seu tipo na plataforma LimeSurvey;

5. Revisão: o questionário criado é revisado pela equipe de desenvolvimento para averiguação do trabalho do programador e não permitir que a pesquisa vá com campos com erros.

6. Publicação: com o questionário pronto ele é divulgado para acesso dos respondentes.

7. Fechamento de campo: assim que a enquete recebe a quantidade desejada de respostas ela é fechada o banco de dados é consolidada e entregue ao cliente.

Das etapas listadas acima as que que mais consomem tempo são justamente as etapas de programação do questionário e revisão do qual se encontram também o maior número de erros. Entre os erros mais comumente encontrados estão erros gramaticais e escolha de tipos incoerentes com a pergunta (fraseologia). Em questionários longos é comum a falta de questões ou respostas presentes no documento. Sendo demonstrado que o maior desgaste do processo eram os erros humanos cometidos nele. A proposta do software do presente trabalho é isolar ao máximo possível a interação do humano com o processo de criação transformando seu papel um de acompanhamento e verificação ao invés de um papel de execução.

\subsection{Proposta}

O software desenvolvido é um serviço web em programado na linguagem Pyhton divido em 3 partes: i) um editor de texto personalizado que permite aos usuários manipular o documento gerado na "elaboração do documento do questionário" marcando os enunciados das questões e suas respostas, ii) um módulo capaz de sugerir tipos para as questões (chamado de sugestor), e iii) uma tela para customização e finalização da enquete.

A ideia por trás do editor está na agilidade de identificação das questões e suas possíveis resposta (para questões fechadas). Foi identificado que os erros em sua grande parte ocorriam quando o programador tinha que transcrever o documento para a plataforma podendo errar a gramática durante a transcrição ou ainda esquecer uma determinada questão. Como o programador não terá que redigir novamente a questão ele estará sujeito a cadastrar a pergunta do modo como ela foi acordada entre as partes. Como insumo deste editor é gerado um arquivo réplica do documento original, porém com as questões e respostas identificadas sendo possível no futuro treinar uma máquina para realizar essa identificação de maneira automatizada.

O sugestor de tipos tira do programador a responsabilidade da escolha apropriada do tipo da questão usando do histórico prévio e da análise do enunciado da questão, através de técnicas de aprendizagem de máquina. O sugestor, então, prediz qual é o tipo mais adequado para cada uma das questões.

A tela de customização vem em sequência para gerar maior flexibilidade ao software permitindo ao usuário mudar ou adicionar uma questão ou resposta, alterar um tipo e fazer a conferência das saídas do software.

\subsubsection{Sugestor de tipos}

O componente de sugestão de tipos é uma árvore de decisão treinada a partir de dados transacionais históricos da base do software LimeSurvey de uma empresa que já tenha utilizado.

O estudo de Pôs estudo inicial foi notificado que apenas 11 tipos são relevantes para a empresa sendo que os demais são apenas derivações destes. Estes tipos são:

- 'mc': "Multiple Choice", tipo de pergunta onde o usuário pode selecionar mais de uma resposta para a pergunta, comumente apresentado como uma sequência de caixas de seleção com uma descritiva.

- 'Ir': "List Radio”, tipo de pergunta onde o usuário tem que selecionar uma única resposta para a resposta dentro de uma lista de opções de botões radio.

- 'ni': "Numerical Input", pergunta onde o usuário deve atribuir um número como resposta;

- 'mni': "Multiple Numerical Input”, pergunta onde o usuário deve atribuir várias respostas numéricas como resposta, exemplo: "Para cada produto a seguir determine uma nota de 1 a 10 visando qualidade.";

- 'arr': “Array”, pergunta onde o usuário tem que atribuir uma ou mais respostas para inúmeros "objetos";

- 'arn': “Array Numérico”, questão semelhante a 'arr' porém aceita como entrada apenas números;

- 'sft': "Small Free Text", questão de texto aberto que aceitam número reduzido de caracteres, usado comumente para inserção de adjetivos;

- 'mft': "Medium Free Text", semelhante a sft porém com número de caracteres aceitos ampliado;

- 'lft': “Large Free Text”, semelhante a sft porém com número de caracteres aceitos largamente ampliado, comumente usado para inserção de textos;

- 'otr': “Outros”, pergunta que não possui tipo identificável;

Com base nos tipos de campos utilizados para respostas os dados foram extraídos da base e processados de modo que se 
apresentassem da seguinte forma: $<<$ sid, code, question, answers, type $>>$, que respectivamente significam o código da enquete, de onde vem a pergunta, o código da questão, o texto da questão, suas respostas e o tipo cadastrado, conforme recorte da base de dados apresentada na Figura 1.

\begin{tabular}{|c|c|c|c|c|c|}
\hline & sid & code & question & answers & type \\
\hline 0 & 987457 & F1 & ALGUÉM NA SUA CASAIFAMILIA TRABALHA COM: Por $\mathrm{f} . .$. & [Agência de Publicidade/ Promoção, Instituto d... & mc \\
\hline 1 & 987457 & $F_{2}$ & Você participou de alguma pesquisa de mercado ... & [Sim, Não] & Ir \\
\hline 3 & 987457 & F4 & Qual é a sua idade? & Nan & ni \\
\hline 13 & 987457 & P1P2 & A PARTIR DESTE MOMENTO VAMOS FALAR SOBRE MARCA... & ['answers: [Conhece', 'Nāo conhece'], 'sub_a... & arr \\
\hline 14 & 987457 & P3P4 & seguintes & Is': "'Comprou alguma vez', & arr \\
\hline
\end{tabular}

Figura 1 - Recorte da base de dados

Nota importante é que a coluna "answers" pode ser apresentada como uma lista quando a pergunta tiver uma lista de possíveis respostas, dicionário se a pergunta for de algum tipo matricial, arr e arn e também pode aparecer como nula quando a pergunta não possui resposta cadastrada como por exemplo nas perguntas de texto aberto $s f t, m f t$ e $l f t$.

\subsubsection{Uso de aprendizagem de máquina no processamento de linguagem natural}

O objetivo primário da aprendizagem de máquina é, antes de tudo, o aprendizado do mapeamento da entrada-saída que é apresentado durante a etapa de treinamento até que um determinado critério seja atendido. De modo geral é utilizada uma medida de erro no qual se compara a resposta gerada pela máquina de aprendizagem com a resposta do conjunto de dados de treinamento (Vapnik (1995), Aguirre, (2004), Bishop (1996)).

O uso de aprendizagem de máquina para utilização no processamento de linguagem natural é muito estudado, uma vez que a capacidade de processamento alinhado com a melhoria da técnica, vem trazendo ótimos resultados, conforme pode ser observado nos estudos: Arroyo-Fernandez et.al. (2019), Hannad et.al. (2019), Das, Abhishek (2019) e Alharbi e de Doncker (2019).

A integração proposta no presente trabalho propõe que as técnicas de aprendizagem de máquina como: árvore de decisão, KNN (K-Nearest Neighbors), classificador perceptrons multi-camadas e Máquina de Vetores de Suporte (SVMs) (Haykin e Engel (2003)).

Foi proposta para o presente trabalho a seguinte configuração:

- Árvore de decisão: Gerada pelo método de árvore randômica, com geração de 300 árvores;.

- MLP Classifier: Foi utilizada uma única camada oculta com 300 perceptrons e a função de ativação para os perceptrons foi a unidade linear retificada;
- SVM: Para cada uma das classes já citadas foram criados respectivamente $47,239,54,572,356,78$, 13, 31, 59, 92 vetores de suporte para cada uma delas. Sendo configurada com penalidade 10, kernel $r b f$, e coeficiente do kernel de 0.01 ;

- KNN: Modelado com valor de vizinhos igual a 3 e a distância utilizada foi a Euclidiana.

Todas as técnicas utilizaram da seguinte proporção $30 \%$ dos valores para teste e $70 \%$ para treinamento e utilizaram de validação cruzada para averiguação dos resultados, assim como verificação das matrizes de confusão de cada modelo.

\section{RESULTADOS ENCONTRADOS}

Com os dados dispostos da forma apresentada na Seção 2.2.2 foi aplicada então a técnica proveniente de processamento de linguagem natural chamada CountVectorizer na coluna questão, de acordo com o trabalho de Eshan e Hasan (2017). Com isso foi possível gerar um vetor para cada questão que a representa contagem de ocorrências de todos os termos utilizados historicamente, podendo assim comparar a similaridade perante os enunciados e estudar a correlação dos usos dos termos com o tipo das perguntas. Também foram criadas mais duas colunas que evidenciam o tipo da resposta.

A concatenação dessas variáveis explicativas geraram 3880 colunas sendo 3788 colunas referentes aos termos utilizados, e com a variável resposta sendo o tipo da pergunta.

Este conjunto de dados foi apresentado a diversos modelos de classificação multiclasse como árvore de decisão, KNN, classificador perceptrons multi-camadas e máquina de vetores de suporte. $\mathrm{O}$ modelo que apresentou a melhor resposta para este conjunto de dados foi a árvore de decisão. A Figura 2 com o modelo dela obtemos a seguinte matriz de confusão, para a seguinte ordem de tipos:

\begin{tabular}{|l|r|r|r|r|r|r|r|r|r|r|}
\hline & arn & arr & Ift & Ir & mc & mft & mni & ni & otr & sft \\
\hline arn & 10 & 16 & 0 & 0 & 0 & 0 & 0 & 0 & 0 & 0 \\
\hline arr & 0 & 116 & 0 & 0 & 0 & 0 & 0 & 0 & 0 & 0 \\
\hline Ift & 0 & 0 & 19 & 0 & 0 & 0 & 0 & 0 & 0 & 15 \\
\hline Ir & 0 & 1 & 0 & 377 & 0 & 0 & 0 & 0 & 1 & 0 \\
\hline mc & 0 & 1 & 0 & 0 & 53 & 113 & 0 & 0 & 0 & 0 \\
\hline mft & 0 & 3 & 0 & 0 & 17 & 5 & 12 & 0 & 0 & 0 \\
\hline mni & 0 & 1 & 0 & 2 & 0 & 0 & 1 & 0 & 0 & 0 \\
\hline ni & 0 & 0 & 0 & 0 & 0 & 0 & 0 & 10 & 0 & 16 \\
\hline otr & 0 & 2 & 0 & 0 & 0 & 0 & 0 & 0 & 21 & 1 \\
\hline sft & 0 & 0 & 1 & 0 & 0 & 0 & 0 & 1 & 0 & 41 \\
\hline
\end{tabular}

Figura 2 - Matriz de confusão para a árvore de decisão. 
A matriz de confusão mostra que a pior precisão do modelo, com $56 \%$, foi o tipo de campo 'sft' onde o modelo pode classificá-lo como sendo 'lft' ou 'ni'. O modelo em geral apresenta uma acurácia média de $86 \%$. Para geração deste modelo foi utilizado o método de floresta randômica sendo gerada 300 árvores para comparação.

A título de comparação, seguem a acurácia obtida a partir dos demais modelos utilizados:

- MLP Classifier: $80 \%$

- SVM: $79 \%$

- $\mathrm{KNN}: 70 \%$

Por se tratar de um trabalho em um estágio ainda inicial, considera-se que o resultado encontrado pela árvore de decisão tenha seja satisfatório o suficiente para considerar a técnica como uma importante ferramenta de automatização de enquetes e formulários de pesquisa. Além disso, não foi possível comparar a técnica proposta com outros benchmarks, uma vez que não existe ainda literatura que trate do tema.

\section{CONSIDERAÇÕES FINAIS E TRABALHOS FUTUROS}

O presente trabalho apresentou uma ferramenta que integra técnicas de aprendizagem de máquina com a automatização de construção de formulários. Esta ferramenta visa minimizar o erro causado por fator humano, uma vez que o resultado da própria aprendizagem foi capaz de gerar os campos necessários com uma acurácia média de $85 \%$, para árvore de decisão.

Por se tratar de um trabalho em um estágio ainda incipiente de pesquisa, considera-se que o resultado encontrado até o momento pela árvore de decisão tenha seja satisfatório o suficiente para considerar a técnica como uma importante ferramenta de automatização de enquetes e formulários de pesquisa online para empresas que utilizam a plataforma LimeSurvey.

Como trabalhos futuros os autores desejam explorar demais técnicas de aprendizagem de máquina assim como aprimorar o que já foi realizado com o processamento de linguagem natural. Além disso, espera-se que demais formulários como Google Forms, por exemplo, possam servir como bases de testes e validação da técnica aqui apresentada.

\section{AGRADECIMENTO}

Os autores gostariam de agradecer à Faculdade Cotemig e ao Offerwise pelo apoio e suporte a este trabalho.

\section{REFERÊNCIAS}

Aguirre, L. (2004). Introdução à Identificação de Sistemas Técnicas Lineares e Não-Lineares Aplicadas a Sistemas Reais, Editora UFMG.

Alharbi, Ahmed Sulaiman M. de Doncker, Elise (2019). Twitter sentiment analysis with a deep neural network:
An enhanced approach using user behavioral information. Cognitive systems research. Volume: 54 Páginas: 50-61

Arroyo-Fernandez, Ignacio., Mendez-Cruz, Carlos-Francisco., Sierra, Gerardo., Torres-Moreno, Juan-Manuel., Sidorov, Grigori (2019) Unsupervised sentence representations as word information series: Revisiting TF-IDF. Computer speech and languages. Volume: 56 Páginas: 107-129

Bishop, C. M. (1996). Neural Networks for Pattern Recognition, 1 edn, Oxford University Press, USA.

Cathrine Jansson-Boyd (2010). Consumer Psychology, McGraw-Hill Education.

Das, Abhishek., Kottur, Satwik., Gupta, Khushi., Singh, Avi., Yadav, Deshraj., Lee, Stefan., Moura, Jose M. F., Parikh, Devi., Batra, Dhruv (2019). Visual Dialog. IEEE transactions on pattern analysis and machine intelligence. Volume: 41 Edição: 5 Páginas: 1242-1256

Eshan, Shahnoor C. e Hasan, Mohammad S (2017). An application of Machine Learning to Detect Abusive Bengali Text. 20th International conference of computer and information. Univ Asia Pacific, Dept Comp Sci \& Engn, Dhaka, bangladesh

Freitas, Henrique., Janissek-Muniz, Raquel., e Moscarola, Jean (2004). Uso da Internet no processo de pesquisa e análise de dados. Associação Nacional de Empresas de Pesquisa, São Paulo.

Hannad, Yaacoub., Siddiqi, Imran., Djeddi, Chawki., El-Kettani, Mohamed El-Youssfi (2019). Improving Arabic writer identification using score-level fusion of textural descriptors. Computer speech and language. Volume: 8 Edição: 3 Páginas: 221-229

Simon Haykin e Paulo Martins Engel (2003). Redes Neurais: Princípios e Prática. Editora Bookman.

Vapnik, V. N. (1995). The nature of statistical learning theory, Springer-Verlag New York, Inc., New York, NY, USA. 